\title{
A Relação entre Indecisão Profissional e Otimismo Disposicional em Adolescentes
}

\author{
Roberta Ramazotti Ferraz de Campos ${ }^{1}$ \\ Ana Paula Porto Noronha \\ Programa de Pós-Graduação Stricto Sensu em Psicologia da Universidade \\ São Francisco, Itatiba, SP, Brasil
}

\begin{abstract}
Resumo
Auxiliar o jovem na compreensão das dificuldades da indecisão profissional para que ele realize uma escolha madura e bem ajustada é um importante objetivo da Orientação Profissional (OP). O presente trabalho pretendeu relacionar indecisão profissional e otimismo em jovens aprendizes e estudantes do Ensino Médio/Técnico. Participaram 250 jovens que frequentam cursos de ensino técnico-profissional, concomitantemente (Grupo 1) ou não com (Grupo 2), o Ensino Médio. Os instrumentos utilizados foram Inventário de Levantamento das Dificuldades da Decisão Profissional (IDDP) e Revised Life Orientation Test Brasil (LOT-R Brasil). Foram observadas correlações negativas de baixa magnitude entre os fatores referentes à indecisão profissional e otimismo. Os grupos de diferenciaram em relação a três fatores do IDDP, sendo que o Grupo 2 obteve médias maiores para esses resultados. Em relação ao sexo, os homens tiveram maior média que as mulheres para dois fatores do IDDP, mostrando tendência a maiores dificuldades no processo de decisão por uma profissão. Os resultados demonstraram que os índices de otimismo foram maiores que os de pessimismo, e que a indecisão profissional está mais presente em indivíduos pessimistas, sugerindo que indivíduos mais seguros estão mais preparados para realizar a escolha por uma profissão.
\end{abstract}

Palavras-chave: Otimismo, indecisão profissional, adolescentes, orientação profissional, psicologia positiva.

\section{The Relationship between Career Indecision and Dispositional Optimism in Adolescents}

\begin{abstract}
Assist young people in understanding the difficulties in career decision for him to perform a mature and well-adjusted choice is an important goal of the Vocational Guidance (VG). This paper intended to relate vocational indecision and optimism in young learners and students of high school/Technical. Participated 250 young people attending courses in technical and vocational education, concomitantly (Group 1) or not (Group 2), the High School. The instruments used were the Inventário de Levantamento das Dificuldades da Decisão Profissional (IDDP) and Revised Life Orientation Test Brasil (LOT-R Brasil). Negative low correlations between factors related to vocational indecision and optimism were observed. The groups differed in relation to three factors IDDP, and Group 2 had higher averages for

Endereço para correspondência: Rua Alexandre Rodrigues Barbosa, 45, Centro, Itatiba, SP, Brasil 13253-231. Fone: (11) 4534-8118. E-mail: roberta.rfcampos@gmail.com e ana.noronha@usf.edu.br As autoras agradecem a Coordenação de Aperfeiçoamento de Pessoal de Nível Superior (CAPES) pelo financiamento da pesquisa de Mestrado da primeira autora, defendida em Agosto/2013, com o título "Indecisão Profissional e Otimismo em Jovens Aprendizes".
\end{abstract}


these results. Regarding gender, men had higher scores than women in two factors of IDDP, scores than women in two factors of IDDP, showing a tendency to greater difficulties in the decision making process by a profession. The results demonstrated that the levels of optimism were higher than the pessimism, and that professional indecision is present in more pessimistic individuals, suggesting that more secure individuals are better prepared to make the choice of a profession.

Keywords: Optimism, career indecision, teens, psychological assessment, vocational guidance, positive psychology.

\section{La Relación entre la Indecisión Professional y el Optimismo Disposicional en Adolescentes}

\section{Resumen}

Ayudar los jóvenes a comprender las dificultades de la indecisión profesional para darse cuenta de una elección madura y bien ajustada es un objetivo importante de la Orientación Profesional (OP). Este estudio tiene como objetivo relacionar la indecisión profesional y el optimismo en jóvenes aprendices y estudiantes de la escuela normal/educación técnica. Participaron 250 jóvenes que asisten a cursos de educación técnica y profesional, junto (Grupo 1) o no (Grupo 2) con la Escuela Secundaria. Los instrumentos utilizados fueron el Inventário de Levantamento das Dificuldades da Decisão Profissional (IDDP) y el Revised Life Orientation Test Brasil (LOT-R Brasil). Se observaron correlaciones bajas y negativas entre los factores relacionados con la indecisión y el optimismo profesional. Los grupos se diferenciaron en relación a tres factores IDDP, y Grupo 2 tuvo los promedios más altos para estos resultados. En relación al sexo, se encontró que los hombres tuvieron puntuaciones más altas que las mujeres en dos factores del IDDP, lo que sugiere una tendencia a mayores dificultades en el proceso de decisión de una profesión. Los resultados mostraron que los niveles de optimismo fueron más altos que los de pesimismo, y que la indecisión profesional está presente en individuos más pesimistas, lo que sugiere que las personas más seguras están mejor preparados para hacer la elección de una profesión.

Palabras clave: Optimismo, indecisión professional, adolescentes, evaluación psicológica, orientación profesional, psicología positiva.

Realizar a escolha por uma profissão ou carreira não é fácil, pois para alguns indivíduos pode estar associada a dificuldades e conflitos, e implica inúmeras opções profissionais. A escolha profissional mais confortável às suas características pessoais e necessidades pode ser selecionada pelo indivíduo a partir do reconhecimento das decisões mais e menos importantes, para que o objetivo desejado seja alcançado (Hutz \& Bardagi, 2006; Levenfus, 2010; Primi et al., 2000).

A Orientação Profissional (OP) tem como principal objetivo o auxílio na compreensão das informações pessoais e profissionais e nas questões que circundam a tomada de decisões. $\mathrm{O}$ reconhecimento das potencialidades dos indivíduos tende a minimizar a indecisão profissional e pode propiciar condições para autorreflexão e aprendizado, visando uma escolha madura e bem-sucedida, além de ter caráter mediador entre indivíduo e suas aspirações na inserção no mercado de trabalho (Ginevra, Nota, Soresi, \& Gati, 2012; Levenfus, 2010; Oliveira \& Neiva, 2013; Primi et al., 2000). Noronha e Ambiel (2008) e Rocha (2010) salientam que os profissionais da área de OP podem fazer o uso de instrumentos diagnósticos na avaliação psicológica desses jovens, a fim de promover a compreensão de suas das necessidades, minimizando a indecisão profissional.

No Brasil, a prática mais tradicional e reconhecida na área de OP é o trabalho com os alunos do Ensino Médio (Sobrosa, Camerin, Santos, \& Dias, 2012). O período de transição do Ensino Médio para o Superior ou para o 
mercado profissional é um momento muito importante para o adolescente. Além de marcar a entrada para a vida adulta, os jovens podem não ter clareza sobre a multiplicidade de profissões, áreas de estudo e cursos, o que pode acarretar conflito na escolha. É nessa perspectiva que se defende a relevância da investigação da indecisão, pois muitas vezes os indivíduos podem não ter clareza da melhor escolha devido ao aumento das opções das atividades profissionais, bem como em razão da falta de preparo para fazê-la (Ginevra et al., 2012; Melo-Silva, Lassance, \& Soares, 2004). As dificuldades da decisão profissional podem ser minimizadas à medida que os indivíduos experimentam a realidade, adquirem mais conhecimentos sobre si e sobre as atividades profissionais e definem um conjunto de interesses em temas cada vez mais específicos (Carvalho \& Taveira, 2012; Primi et al., 2000).

O modelo taxonômico hierárquico proposto por Gati, Krausz e Osipow (1996) se organiza a partir da divisão das dificuldades de escolha, tendo como referência o processo geral da tomada de decisão. Assim, quando o indivíduo necessita tomar uma decisão, há diversas alternativas de seleção e inúmeros aspectos a serem considera- dos para comparar, avaliar e, finalmente, fazer a opção de maior utilidade na percepção de suas preferências e características. As dificuldades existentes antes e durante o processo de escolha profissional são organizadas em dois grupos principais, sendo que o primeiro se refere à imaturidade geral para decidir-se profissionalmente ('Antes de iniciar o processo') e o segundo envolve as dificuldades vividas durante o processo de escolha ('Durante o processo').

As informações anteriores ao início do processo de tomada de decisão de carreira englobam, basicamente, a insuficiência de informações sobre si mesmo e sobre as áreas profissionais. Já a falta de informação e conhecimento sobre as etapas envolvidas e as preferências e capacidades do indivíduo para a seleção de opções estão no grupo sobre o decorrer do processo de tomada de decisão (Gati et al., 1996). Mais recentemente, Ginevra et al. (2012) fizeram novas projeções a respeito das dificuldades na tomada de decisão de carreira, sugerindo três grupos de fatores que podem contribuir com a indecisão. As informações sobre os dois modelos estão dispostas na Tabela 1. Ao observar a Tabela 1, nota-se que as diferenças entre as duas taxonomias estão apenas na organização dessas dificuldades.

Tabela 1

Modelos Taxonômicos das Dificuldades da Decisão de Carreira

\begin{tabular}{|c|c|c|c|c|}
\hline \multicolumn{2}{|c|}{ Gati et al. (1996) } & \multirow{2}{*}{\multicolumn{3}{|c|}{$\begin{array}{l}\text { Ginevra et al. (2012) } \\
\text { Durante o processo }\end{array}$}} \\
\hline Antes de iniciar o processo & Durante o processo & & & \\
\hline $\begin{array}{l}\text { Falta de motivação, } \\
\text { indecisão, mitos } \\
\text { disfuncionais e falta } \\
\text { de conhecimento }\end{array}$ & $\begin{array}{l}\text { Falta de informação } \\
\text { e/ou informação inconsistente }\end{array}$ & $\begin{array}{c}\text { Falta de } \\
\text { informação } \\
\text { sobre si mesmo, } \\
\text { as profissões } \\
\text { e o mundo } \\
\text { do trabalho }\end{array}$ & $\begin{array}{l}\text { Consequências } \\
\text { sobre a tomada de } \\
\text { decisão }\end{array}$ & $\begin{array}{c}\text { Falta de } \\
\text { autoconhecimento } \\
\text { generalizado }\end{array}$ \\
\hline $\begin{array}{l}\text { Problemas enfrentados } \\
\text { antes do engajamento na } \\
\text { escolha por uma profissão } \\
\text { específica, no que se refere } \\
\text { à falta de informações } \\
\text { sobre si mesmo e sobre } \\
\text { as áreas profissionais, } \\
\text { bem como as expectativas } \\
\text { irracionais perante } \\
\text { a possibilidade da escolha } \\
\text { e a falta de conhecimento } \\
\text { sobre o que deve ser } \\
\text { efetivado para que } \\
\text { o processo aconteça }\end{array}$ & $\begin{array}{l}\text { Problemas enfrentados } \\
\text { durante a tomada de decisão } \\
\text { de carreira em relação às } \\
\text { etapas envolvidas, devido a } \\
\text { falta de informações sobre si } \\
\text { mesmo, suas preferências e } \\
\text { suas capacidades individuais } \\
\text { de percepção do presente } \\
\text { em relação ao futuro, como } \\
\text { também em relação às próprias } \\
\text { profissões e as preferências } \\
\text { incompatíveis com aquilo } \\
\text { que deseja e/ou com o que os } \\
\text { outros sugerem }\end{array}$ & $\begin{array}{c}\text { Visões } \\
\text { pessimistas } \\
\text { sobre } \\
\text { o processo } \\
\text { de tomada } \\
\text { de decisão, } \\
\text { o mundo } \\
\text { do trabalho } \\
\text { e o controle } \\
\text { pessoal } \\
\text { diante das } \\
\text { inúmeras } \\
\text { possibilidades } \\
\text { de escolha }\end{array}$ & $\begin{array}{c}\text { Ansiedade } \\
\text { e incerteza } \\
\text { sobre o processo } \\
\text { de escolha } \\
\text { e seus possíveis } \\
\text { resultados } \\
\text { para sua vida } \\
\text { profissional } \\
\text { e pessoal }\end{array}$ & $\begin{array}{c}\text { Problemas } \\
\text { enfrentados } \\
\text { em relação ao } \\
\text { autoconhecimento, } \\
\text { devido à ansiedade } \\
\text { generalizada, } \\
\text { autoestima, } \\
\text { identidade } \\
\text { profissional } \\
\text { não definida } \\
\text { e conflitos } \\
\text { interpessoais }\end{array}$ \\
\hline
\end{tabular}


Deste modo, na proposta de Ginevra et al. (2012) as três categorias estão inseridas no grupo 'Durante o Processo', existente no primeiro trabalho. Este grupo abrange desde a falta de informação sobre si mesmo e sobre as ocupações, até as informações não confiáveis que levam o indivíduo a terem conflitos internos e externos para com a tomada de decisão. O modelo proposto por Gati et al. (1996), referenciou uma série de instrumentos de avaliação, dentre os quais o Inventário de Levantamento das Dificuldades da Decisão Profissional (Primi et al., 2000), utilizado no presente estudo.

No que respeita as dificuldades para a escolha, Hutz e Bardagi (2006) salientam que o processo de Orientação Profissional deve promover o autoconhecimento e o conhecimento da realidade do mundo do trabalho para diminuir as indecisões profissionais. Gati e Tal (2008) acrescentam que as escolhas de carreira estão entre as decisões mais importantes e expressivas na vida dos indivíduos, além de ter implicações nos âmbitos social, familiar e pessoal. Deste modo, quando esse processo é bem encaminhado, o indivíduo tende a adquirir um recurso psicológico de enfrentamento de situações complexas ou acontecimentos ameaçadores. É nesse ensejo que se fazem pertinentes as concepções da Psicologia Positiva, pois segundo Seligman (2002) as virtudes e os pontos fortes dos indivíduos funcionam como fatores protetivos de doenças mentais e físicas, e consequentemente, podem ser úteis para minimizar dificuldades ao longo da vida pessoal e profissional.

Contreras e Esguerra (2006) e Seligman (2002) asseveram que as forças e virtudes humanas, além de suas motivações e capacidades, melhoram a qualidade de vida, tendem a reduzir a incidência de psicopatologias e oportunizam vidas mais produtivas e felizes. Entretanto, o trabalho com este movimento científico em conjunto com outras áreas tão importantes quanto ele, é necessário para que os indivíduos tenham um acompanhamento pleno na ajuda à resolução de seus problemas pessoais e profissionais.

Dentre os construtos defendidos pela Psicologia Positiva destaca-se o otimismo disposicio- nal, definido por Scheier e Carver (1985) como a tendência estável das pessoas acreditarem que coisas boas acontecerão, em vez de coisas ruins, antes mesmo de se decidirem sobre a forma como enfrentarão diferentes situações. Carver e Scheier (2005) salientam que a pessoa deve ter confiança suficiente para enfrentar as situações de adversidade para se mover para a ação e continuar a empregar esforços para alcançar seu objetivo, encarando suas falhas e fracassos como temporários, e não como incapacidade pessoal. $\mathrm{O}$ pessimismo também é referenciado nesta teoria, pois as pessoas podem variar de muito otimistas a muito pessimistas, embora geralmente ocupem um lugar intermediário. Portanto, ao contrário das pessoas otimistas, as pessimistas como não conseguem esperar por resultados concretos em determinado momento de suas vidas, tendem a pensar que o pior pode acontecer, mesmo sem saber como enfrentarão tais situações (Scheier \& Carver, 1985, 1993).

Para Carver, Scheier e Segerstrom (2010), o conceito de otimismo disposicional da vida no contexto educacional tem sido estudado e relacionado à capacidade de adaptação escolar. Os autores apontaram que o otimismo e o bem-estar são fatores preditivos de menor sofrimento em alunos ingressantes na universidade. Após aplicar o Revised Life Orientation Test (LOT-R) em estudantes quando chegavam ao campus universitário e novamente ao final do semestre, constataram que a taxa de abandono do curso superior para pessimistas foi de $30 \%$. Para os estudantes considerados otimistas a taxa foi de apenas $15 \%$. Nessa mesma direção, Viloria, Yáñes e Vañó (2010) ressaltaram que estudar os ambientes educacionais do ponto de vista da Psicologia Positiva é essencial, não apenas para a prática de educadores e psicólogos, como também para alunos de quaisquer faixas etárias, pois facilitar o bem-estar em ambientes escolares é o caminho para um melhor desempenho acadêmico e profissional.

Evidências empíricas apontam que otimismo e pessimismo possuem efeitos importantes no bem-estar físico e mental (Carver et al., 2010; Scheier, Carver, \& Bridges, 2001; Shane \& Sny- 
der, 2003), bem como na performance acadêmica e profissional (Aspinwall \& Taylor, 1992; Long, 1993; Lyubomirsky, Tkach, \& DiMatteo, 2006), e também na transição para o mercado de trabalho (Rashid, Arif, \& Hussain, 2012; Yun-Jeong, \& Kevin, 2013). Ainda sobre otimismo, autores encontraram associações com esperança e autoestima, indicando que indivíduos otimistas, tendem a ser mais persistentes em seus objetivos, bem como se sentem mais seguros na tomada de decisão por uma escolha ou carreira que lhes traga sucesso e satisfaça suas necessidades financeiras (Alarcon, Bowling, \& Khazon, 2013; Bastianello, Pacico, \& Hutz, 2014), além de que indivíduos otimistas aumentam suas chances de sucesso pessoal e profissional (Andersson, 2012; Boehm \& Lyubomirsky, 2008; Harpaz-Itay \& Kaniel, 2012; Little, 2012).

Em relação à indecisão profissional, estudos demonstram que indivíduos mais maduros têm menos indecisão e tendem a se comprometer mais com suas escolhas, o que contribui positivamente para a tomada de decisão por uma profissão ou carreira (Proyer, Sidler, Weber, \& Ruch, 2012); que os homens mais jovens, que vivenciam conflitos com familiares e amigos, e cujos pais não possuem curso de graduação são mais indecisos para realizar escolhas de cunho profissional e acadêmico (Choi et al., 2011; Stoltz \& Young, 2012), e que adolescentes filhos de pais desempregados manifestam crenças negativas acerca da transição para o mercado de trabalho e consequentemente, níveis mais elevados de indecisão profissional (Faria, 2013; Magalhães, Alvarenga, \& Teixeira, 2012).

Exatamente sobre a relação entre os construtos indecisão profissional e otimismo, Creed, Patton e Bartrum (2002), observaram que não houve diferenças entre idade e sexo em jovens do Ensino Médio. Os instrumentos utilizados foram o Revised Life Orientation Test (LOT-R), a Career Development Inventory (CDI-A) e a Career Decision Scale (CDS), que mensuram o otimismo, a maturidade de carreira e tomada de decisão de carreira, respectivamente. Adicionalmente, os autores encontraram correlações moderadas e positivas entre otimismo e desenvolvimento de carreira, o que permitiu compreender que altos escores em otimismo estão associados com maior planejamento de carreira. Entre pessimismo e avanço para o desenvolvimento de carreira, foram encontradas correlações moderadas e negativas, sugerindo que altos níveis de pessimismo estão associados a baixos níveis de avanço para o desenvolvimento de estratégias para a escolha de uma carreira.

Enfim, considerando a relevância das pesquisas citadas, ressalta-se que os estudos sobre OP e Psicologia Positiva devem ser continuados, pois os conjuntos das qualidades humanas são importantes fatores protetivos, ao mesmo tempo em que ajudam os indivíduos em suas escolhas pessoais, acadêmicas e profissionais, minimizando suas indecisões, facilitando o bom funcionamento e as potencialidades do ser humano. $\mathrm{O}$ presente estudo pretendeu relacionar indecisão profissional e otimismo em jovens aprendizes e estudantes do Ensino Médio/Técnico, utilizando os instrumentos Inventário de Levantamento das Dificuldades da Decisão Profissional (IDDP) e LOT-R Brasil, a versão brasileira do Revised Life Orientation Test (LOT-R). Assim, supõe-se que indecisão profissional e otimismo estejam negativamente relacionados.

\section{Método}

\section{Participantes}

Fizeram parte do presente estudo 250 alunos do sétimo ao nono ano do Ensino Fundamental, e do primeiro ao terceiro ano do Ensino Médio, de duas cidades do interior do estado de São Paulo. Os jovens pertencentes ao Grupo 1 frequentavam, no momento da pesquisa, uma escola municipal de ensino profissionalizante que oferece cursos técnicos (simultaneamente com o Ensino Médio), e totalizavam 116 participantes, sendo $61,2 \%$ do feminino, com idades entre 14 e 18 anos $(M=16,02 ; D P=0,978)$. Estes alunos estavam envolvidos em um programa de Orientação Profissional vinculado a um amplo projeto de pesquisa, no qual responderam a instrumentos de avaliação psicológica de interesse profissional, habilidades, criatividade, características de personalidade, afeto, otimismo, esperança e indecisão profissional. 
O Grupo 2, frequentava uma instituição de ensino-técnico profissional separadamente do Ensino Fundamental ou Médio, e contava com 134 participantes, dos quais $50,7 \%$ eram do sexo masculino, com idades variando entre 15 e 17 anos, com média de 15,60 $(D P=0,507)$. Em relação ao ano, $0,8 \%$ frequentavam o sétimo, $2,4 \%$ o oitavo e 4,8\% o nono ano do Ensino Fundamental. No Ensino Médio, 36,0\% estavam no primeiro ano, $35,6 \%$ no segundo, e $20,4 \%$ no terceiro ano. O programa de aprendizagem para o trabalho que o Grupo 2 estava inserido foi elaborado de acordo com a Lei Federal no 0.097/2000 (Ministério do Trabalho e Emprego [MTE], 2000), denominada Lei da Aprendizagem, de modo que todas as empresas de médio e grande e porte devem contratar um número de aprendizes no seu quadro de funcionários (mínimo de $5 \%$ e máximo de $15 \%$ ) cujas funções demandem formação profissional.

\section{Instrumentos}

IDDP - Inventário de Levantamento das Dificuldades da Decisão Profissional (Primi et al., 2000). Constituído de 81 itens, avalia as várias facetas da dificuldade da decisão profissional. Primeiramente, 17 fatores foram encontrados, e a partir deles, quatro fatores principais foram agrupados hierarquicamente, a saber: Fator 1 Insegurança e falta de informação $(\alpha=0,89)$, que avalia a insegurança, falta de informações e obstáculos financeiros; Fator 2 - Ênfase na busca de prestígio e retorno financeiro $(\alpha=0,79)$ correspondente a componentes econômicos de prestígios financeiros; Fator 3 - Imaturidade para a escolha $(\alpha=0,70)$ que mede as características de indisposição na tomada de decisões; e finalmente, o Fator 4 - Conflitos com pessoas significativas $(\alpha=0,71)$ que revela a desaprovação do meio quanto à escolha (Primi et al., 2000).

LOT-R Brasil - Revised Life Orientation Test Brasil (Bastianello et al., 2014). É um teste de autorrelato para mensurar o otimismo disposicional, sendo uma versão reduzida e revisada do Life Orientation Test (LOT; Scheier \& Carver, 1985). Contém 10 itens em uma escala tipo Likert que varia de discordo plenamente - 1, até concordo plenamente - 5. São três afirmativas sobre otimismo (itens 1, 4 e 10), três sobre pessimismo (itens 3,7 e 9 ) e quatro itens distratores que não são computados $(2,5,6,8)$. O LOT-R Brasil apresenta boa consistência interna $(\alpha=$ $0,77)$ e suas correlações teste-reteste são de 0,68 a 0,79 para intervalos de 4 a 28 meses (Bastianello, 2011; Bastianello et al., 2014). Ademais, o instrumento apresentou uma estrutura unidimensional, sendo que a presença de um único fator explicou aproximadamente $48 \%$ da variância total dos dados, estando de acordo com os achados do estudo original (Bastianello et al., 2014; Scheier, Carver, \& Bridges, 1994).

\section{Procedimento}

Com a autorização das instituições de ensino, e em seguida a aprovação do projeto pelo Comitê de Ética em Pesquisa de uma Universidade do Estado de São Paulo, sob o número 103.695, foram obtidas as autorizações dos pais ou responsáveis por meio do Termo de Consentimento Livre e Esclarecido, enviado a eles por intermédio dos próprios alunos. Em seguida, explicou-se aos jovens sobre o sigilo e a confidencialidade das informações, os objetivos do estudo, a forma de participação, como também a ausência de riscos ou danos decorrentes da participação ou não.

Os instrumentos foram aplicados coletivamente nas salas de aulas das instituições, com aproximadamente 48 alunos por sala. A aplicação iniciou-se pelo rapport, seguido da distribuição dos instrumentos para cada participante, e posteriormente, as instruções foram dadas, solicitando que as dúvidas fossem esclarecidas apenas antes do início do preenchimento dos testes. O processo de aplicação dos dois instrumentos teve duração aproximada de 45 minutos, sendo que, primeiramente, foi aplicado o IDDP e em seguida, o LOT-R Brasil.

\section{Resultados}

O objetivo do presente estudo foi relacionar indecisão profissional e otimismo em jovens aprendizes e estudantes do Ensino Médio/Técnico. A seguir encontram-se os valores mínimos e máximos, bem como as médias e os respecti- 
vos desvios padrão para cada um dos fatores do IDDP na Tabela 2. Tendo em vista que o Fator 1 - Insegurança e falta de informação contém sete subfatores, o Fator 2 - Ênfase na busca de prestígio e retorno financeiro tem quatro subfa- tores, e os fatores 3 - Imaturidade para escolha e 4 - Conflitos com pessoas significativas possuem três subfatores, ressalta-se que os valores são resultantes de ponderação, a fim de permitir a comparação do número desigual dos itens.

Tabela 2

Estatística Descritiva do IDDP e do LOT-R Brasil (N=250)

\begin{tabular}{ccccc}
\hline & Mínimo & Máximo & Média & Desvio Padrão \\
\hline Insegurança e falta de informação & 1,27 & 5,66 & 3,36 & 0,92 \\
Ênfase na busca de prestígio e retorno financeiro & 1,33 & 6,63 & 4,13 & 0,95 \\
Imaturidade para a escolha & 1,00 & 6,25 & 2,79 & 1,10 \\
Conflitos com pessoas significativas & 1,17 & 5,08 & 2,92 & 0,89 \\
Otimismo & 5,00 & 15,00 & 12,24 & 2,28 \\
Pessimismo & 3,00 & 15,00 & 6,30 & 2,64 \\
\hline
\end{tabular}

Ao observar na Tabela 2, nota-se que o fator Ênfase na busca de prestígio e retorno financeiro apresentou maior média $(M=4,13 ; D P=0,95)$. Esse resultado revela a existência de uma tendência à valorização do aspecto econômico e de prestígio social que algumas profissões podem proporcionar aos indivíduos. Além disso, infere-se que os jovens da amostra tem a expectativa de que a profissão resolva seus problemas financeiros e sociais. Já o fator Imaturidade para a escolha apresentou menor média $(M=2,79)$, embora com o maior desvio padrão $(D P=1,10)$, o que indica maior variabilidade de respostas. Os demais fatores, Insegurança e falta de informação e Conflitos com pessoas significativas, apresentaram média de 3,36 $(D P=0,92)$ e 2,92 ( $D P$ $=0,89)$, respectivamente.
Em relação ao Otimismo, coube a este a maior média, indicando que esses jovens possuem uma tendência estável de acreditar que coisas boas acontecerão, em vez de coisas ruins. Infere-se que os indivíduos da amostra estão mais preparados para enfrentar as adversidades da vida com sucesso, antes mesmo de se decidirem sobre a forma como enfrentarão essas situações. A menor média em Pessimismo permite sugerir que a expectativa de resultados negativos diante de situações difíceis é menor na amostra estudada.

Foi realizada a correlação de Pearson para verificar as relações entre os instrumentos IDDP e LOT-R Brasil, utilizados no presente estudo. Os resultados estão expostos na Tabela 3.

\section{Tabela 3}

Correlação de Pearson entre os Fatores do IDDP e Otimismo e Pessimismo do LOT-R Brasil

\begin{tabular}{llcccc}
\hline & & $\begin{array}{c}\text { Insegurança e falta } \\
\text { de informação }\end{array}$ & $\begin{array}{c}\text { Ênfase na busca de prestígio } \\
\text { e retorno financeiro }\end{array}$ & $\begin{array}{c}\text { Imaturidade } \\
\text { para a escolha }\end{array}$ & $\begin{array}{c}\text { Conflitos com pessoas } \\
\text { significativas }\end{array}$ \\
\hline Otimismo & $r$ & $-0,24^{*}$ & $0,20^{*}$ & $-0,11$ & $-0,06$ \\
& $p$ & 0,001 & 0,001 & 0,098 & 0,359 \\
Pessimismo & $r$ & $0,33^{*}$ & $0,21^{*}$ & $0,30^{*}$ & 0,041 \\
& $p$ & 0,001 & 0,001 & 0,001 & 0,523 \\
\hline
\end{tabular}

Nota. * Correlações significativas ao nível de 0,01 . 
Observa-se na Tabela 3 que os resultados revelaram que associações estatisticamente significativas, embora de baixa magnitude, entre três fatores do IDDP e pessimismo. Mais especialmente, o fator Insegurança de falta de informação do IDDP se correlacionou positivamente com pessimismo $(r=0,33)$, sugerindo que os indivíduos da amostra mais inseguros para realizarem a escolha de uma profissão ou carreira tendem a ser mais pessimistas em relação ao futuro profissional. No entanto, possivelmente essa relação é permeada por outras variáveis, já que há que se considerar que o coeficiente entre insegurança e pessimismo foi baixo.

$O$ fator Imaturidade para a escolha do IDDP também se correlacionou positivamente com pessimismo $(r=0,30)$. Assim, há relação, ainda que de baixa magnitude, entre a imaturidade para realizar uma escolha e pessimismo, permitindo compreender que pessoas imaturas sustentam expectativas negativas de êxito e não realização das opções de profissões ou carreiras a serem escolhidas. Ainda sobre o pessimismo, o fator Ênfase na busca de prestígio e retorno financeiro do IDDP, obteve correlação positiva, porém de baixa magnitude, $(r=0,21)$, aventando a hipótese de que o jovem tende a buscar algum prestígio pessoal ou social e retorno financeiro. Ademais, já que se sente pessimista diante da escolha a ser realizada, e por não ter confiança suficiente de que a escolha dará certo, o jovem não emprega esforços para alcançar sua meta.

No que se refere às correlações dos fatores do IDDP com otimismo do LOT-R, apenas em dois fatores houve significância, embora as magnitudes tenham sido baixas. $\mathrm{O}$ fator Insegurança e falta de informação gerou correlação negativa e significativa de baixa magnitude $(r=-0,24)$, o que permite compreender que os indivíduos da amostra mais otimistas tendem a ter menos insegurança sobre si mesmo e suas preferências. Por fim, o fator que se correlacionou positivamente com otimismo, foi Ênfase na busca de prestígio e retorno financeiro $(r=0,20)$.

Visando analisar as possíveis diferenças de médias entre os grupos de participantes, quais sejam, Grupo 1 (jovens que frequentam o ensino médio e técnico-profissional conjuntamente) e 2 (jovens que trabalham e estudam - aprendizes), a seguir são apresentados os resultados por meio do teste $t$ de Student. Resultados significativos foram encontrados para três fatores do IDDP e para otimismo do LOT-R Brasil.

Os grupos se diferenciaram significativamente em relação aos fatores Ênfase na busca de prestígio e retorno financeiro $(t=-2,906$; $p=0,004)$, Imaturidade para a escolha $(t=-5,106$; $p=0,001)$, Conflitos com pessoas significativas $(t=-4,915 ; p=0,001)$ e Otimismo $(t=-2,024$; $p=0,044$ ), de modo que o Grupo 2 (jovens que estudam e trabalham - aprendizes) teve médias maiores. Com estes dados, infere-se que os jovens pertencentes ao Grupo 2 tendem a escolher profissões que possam lhes trazer status social e financeiro. No que se refere ao Otimismo, é possível sugerir que os indivíduos desse grupo quando se deparam com adversidades, tal como a indecisão quanto à escolha de uma profissão, tendem a encarar o processo de tomada de decisão pelo lado positivo.

Com o objetivo de analisar as possíveis diferenças entre os sexos, foi realizado o teste $t$ de Student, tomando como referência o grupo de participantes. Primeiramente, são expostas as diferenças entre os fatores do IDDP e otimismo e pessimismo do LOT-R Brasil para o Grupo 1 (jovens que frequentam o ensino médio e técnico-profissional conjuntamente), e em seguida, para o Grupo 2 (jovens que estudam e trabalham - aprendizes). Os resultados estão na Tabela 4.

A Tabela 4 mostra que os meninos se diferenciaram em dois dos quatro fatores do IDDP, quais sejam, Imaturidade para a escolha $(t=2,781$; $p=0,006)$ e Conflitos com pessoas significativas $(t=3,200 ; p=0,002)$. Os dados sugerem que para essa amostra o sexo masculino é mais imaturo para tomar a decisão de uma profissão. Também é possível compreender que para eles falta organização para que a tomada de decisão por uma profissão ou carreira aconteça. Ainda na Tabela 4 , os dados revelam que para os sexos não foram encontradas diferenças significativas entre otimismo e pessimismo LOT-R Brasil. 
Tabela 4

Médias, Desvios Padrão e Valores de t e p por Sexo para o Grupo 1

\begin{tabular}{|c|c|c|c|c|c|c|}
\hline & Sexo & $N$ & $M$ & $D P$ & $t$ & $p$ \\
\hline \multirow{2}{*}{ Insegurança e falta de informação } & Masculino & 45 & 3,34 & 0,90 & \multirow{2}{*}{$-0,028$} & \multirow{2}{*}{0,978} \\
\hline & Feminino & 71 & 3,35 & 1,03 & & \\
\hline \multirow{2}{*}{$\begin{array}{c}\text { Ênfase na busca de prestígio } \\
\text { e retorno financeiro }\end{array}$} & Masculino & 45 & 4,05 & 0,81 & \multirow{2}{*}{0,953} & \multirow{2}{*}{0,343} \\
\hline & Feminino & 71 & 3,90 & 0,96 & & \\
\hline \multirow[b]{2}{*}{ Imaturidade para a escolha } & Masculino & 45 & 2,74 & 1,14 & \multirow[b]{2}{*}{2,781} & \multirow[b]{2}{*}{0,006} \\
\hline & Feminino & 71 & 2,23 & 0,84 & & \\
\hline \multirow[b]{2}{*}{ Conflitos com pessoas significativas } & Masculino & 45 & 2,90 & 0,74 & \multirow[b]{2}{*}{3,200} & \multirow[b]{2}{*}{0,002} \\
\hline & Feminino & 71 & 2,47 & 0,63 & & \\
\hline \multirow[b]{2}{*}{ Otimismo } & Masculino & 45 & 11,51 & 2,71 & \multirow[b]{2}{*}{$-1,494$} & \multirow[b]{2}{*}{0,138} \\
\hline & Feminino & 71 & 12,20 & 2,20 & & \\
\hline \multirow{2}{*}{ Pessimismo } & Masculino & 45 & 6,46 & 2,80 & \multirow{2}{*}{0,754} & \multirow{2}{*}{0,453} \\
\hline & Feminino & 71 & 6,08 & 2,54 & & \\
\hline
\end{tabular}

Tabela 5

Médias, Desvios Padrão e Valores de t e p por Sexo para o Grupo 2

\begin{tabular}{|c|c|c|c|c|c|c|}
\hline & Sexo & $N$ & $M$ & $D P$ & $t$ & $p$ \\
\hline \multirow{2}{*}{ Insegurança e falta de informação } & Masculino & 68 & 3,47 & 0,92 & \multirow{2}{*}{1,144} & \multirow{2}{*}{0,152} \\
\hline & Feminino & 66 & 3,25 & 0,84 & & \\
\hline \multirow{2}{*}{$\begin{array}{c}\text { Enfase na busca de prestígio } \\
\text { e retorno financeiro }\end{array}$} & Masculino & 68 & 4,45 & 0,93 & \multirow{2}{*}{2,024} & \multirow{2}{*}{0,045} \\
\hline & Feminino & 66 & 4,12 & 0,95 & & \\
\hline \multirow{2}{*}{ Imaturidade para a escolha } & Masculino & 68 & 3,47 & 1,04 & \multirow{2}{*}{4,192} & \multirow{2}{*}{0,001} \\
\hline & Feminino & 66 & 2,73 & 1,00 & & \\
\hline \multirow{2}{*}{ Conflitos com pessoas significativas } & Masculino & 68 & 3,50 & 0,91 & \multirow{2}{*}{4,151} & \multirow{2}{*}{0,001} \\
\hline & Feminino & 66 & 2,90 & 0,91 & & \\
\hline \multirow{2}{*}{ Otimismo } & Masculino & 68 & 12,33 & 2,23 & \multirow{2}{*}{$-0,971$} & \multirow{2}{*}{0,333} \\
\hline & Feminino & 66 & 12,70 & 2,03 & & \\
\hline \multirow{2}{*}{ Pessimismo } & Masculino & 68 & 6,67 & 2,75 & \multirow{2}{*}{0,516} & \multirow{2}{*}{0,161} \\
\hline & Feminino & 66 & 6,03 & 2,54 & & \\
\hline
\end{tabular}


De igual modo para o Grupo 2, os resultados revelaram diferenças significativas com médias maiores para os homens nos mesmos fatores do IDDP, citados anteriormente, além de Ênfase na busca de prestígio e retorno financeiro. Os dados estão na Tabela 5.

Pelos valores expostos na Tabela 5, os fatores Ênfase na busca de prestígio e retorno financeiro $(t=2,024 ; p=0,045)$, Imaturidade para a escolha, $(t=4,192 ; p=0,001)$ e Conflitos com pessoas significativas $(t=4,151 ; p=0,001)$, o sexo masculino apresentou maior média. As informações mostram que esses jovens tendem a buscar algum prestígio pessoal ou social e retorno financeiro, devido à falta de estratégias para obter as informações necessárias referentes às profissões e ao processo de tomada de decisão.

\section{Discussão}

O presente estudo pretendeu relacionar indecisão profissional e otimismo em jovens aprendizes e estudantes do Ensino Médio/Técnico, bem como analisar as diferenças de médias em relação ao sexo e grupo de participantes. Os resultados obtidos demonstraram que o fator Ênfase na busca de prestígio e retorno financeiro, do IDDP, foi o mais valorizado. Este dado está de acordo com o estudo de Melo-Silva, Santos, Palma e Duarte (2007), que identificaram em 26 meninas com idades entre 16 e 18 anos, o desejo em escolher uma profissão ou carreira que lhes satisfaça financeiramente, em primeiro lugar. Os autores relatam que neste momento tão complexo de suas vidas, os jovens tendem a optar por profissões que englobam fatores sociais e econômicos, por considerarem que esta é uma escolha que solucionará todos os seus problemas. Também Macêdo, Alberto e Araújo (2012) identificaram que o interesse em escolher um curso superior está associado à ideia de garantia de inserção no mundo do trabalho, e possivelmente segurança profissional e financeira. Em contrapartida, o fator Conflitos com pessoas significativas foi o menos pontuado, indicando que os jovens da amostra não experimentam desaprovação do meio quanto à escolha (Primi et al., 2000).
Os índices de otimismo foram maiores que de pessimismo, sugerindo que a maior parte da amostra se dispõe e encarar as complexidades da vida com sucesso. Vários autores encontraram este dado em suas pesquisas (Carver \& Scheier, 1999, 2005; Carver et al., 2010; Choi et al., 2011; Hartley, 2009; Scheier \& Carver, 1985, 1993; Scheier et al., 2001; Shane \& Snyder, 2003; Stoltz \& Young; 2012; dentre outros), além de que indivíduos otimistas têm confiança suficiente para se mover para a ação e continuar o emprego de esforços em momentos adversos (Bastianello et al., 2014; Proyer et al., 2012).

No que se refere às correlações entre os instrumentos IDDP e LOT-R Brasil, houve correlação negativa e significativa de baixa magnitude entre Insegurança e falta de informação e otimismo. O dado revela que esses indivíduos se sentem seguros e mais preparados para a tomada de decisão de uma profissão, inferindo-se que são mais otimistas na realização de uma escolha mais ajustada. O fator Ênfase na busca de prestígio e retorno financeiro se correlacionou positivamente com baixa magnitude com otimismo. Assim, supõe-se que os estudantes mais otimistas acreditam que escolherão profissões que lhes deem sucesso pessoal e financeiro (Carver \& Scheier, 1999, 2005; Carver et al., 2010).

No que se refere às correlações entre os fatores do IDDP e pessimismo, três foram significativas. A primeira, positivamente com o fator Insegurança e falta de informação, indicou que os jovens que se sentem inseguros e despreparados para a realização de escolha são mais pessimistas para com o processo de tomada de decisão. Isto pode ocorrer devido à falta de estratégias para a obtenção de informações sobre si mesmo e sobre as profissões, bem como à complexidade de preferências relacionadas aos interesses e habilidades dos indivíduos para tais ocupações (Andersson, 2012; Bastianello et al., 2014; Boehm \& Lyubomirsky, 2008; Harpaz-Itay \& Kaniel, 2012).

A relação entre o fator Imaturidade para escolha e pessimismo também foi constatada, embora de baixa magnitude, permitindo compreender que os jovens imaturos são mais pessimistas em relação ao seu futuro profissional. 
Neste caso, as expectativas negativas de êxito e de não realização das opções profissionais fazem com que o jovem sinta ainda mais dificuldade em optar por alguma ocupação. Por fim, a última correlação positiva, e ainda de baixa magnitude, foi encontrada entre pessimismo e o fator Ênfase na busca de prestígio e retorno financeiro. Com este dado infere-se que a busca pelo sucesso social e financeiro pode ser alcançado fazendo a escolha por uma profissão ou carreira que ofereça status, já que os jovens, quanto mais pessimistas, mais tendem a buscar o prestígio social e financeiro (Carver \& Scheier, 1993; Primi et al., 2000). Contudo, é importante ressaltar que como a magnitude foi baixa, há que se considerar que outras variáveis podem permear essas relações.

Nas diferenças de médias entre os grupos, em três fatores houve diferenças significativas, com média maior para o Grupo 2 (jovens que trabalham e estudam), quais sejam, Ênfase na busca de prestígio e retorno financeiro, Imaturidade para a escolha, e Conflitos com pessoas significativas. Os dados supracitados corroboram os achados de Primi e cols. (2000), que revelaram diferenças significativas para o fator Imaturidade para a escolha em jovens trabalhadores. É possível refletir na direção de que os jovens que trabalham e estudam (Grupo 2), embora experimentem mais imaturidade para com a escolha, sentem-se mais otimistas em relação à futura profissão por já estarem inseridos no mercado de trabalho.

Em relação ao sexo, as análises foram rodadas separadamente para os grupos. As diferenças significativas para o sexo, para Grupo 1 , foram encontradas nos fatores Imaturidade para a escolha e Conflitos com pessoas significativas. Os meninos obtiveram maiores médias nos dois fatores supracitados, dados estes corroborados nos estudos de Hartley (2009). Já para o Grupo 2, diferenças significativas foram encontradas para três fatores do IDDP. No que se refere ao otimismo e pessimismo, não foram encontradas diferenças significativas para os sexos, tanto para o Grupo 1, quanto para o Grupo 2 (Bastianello, 2011; Creed et al., 2002), o que nos permite inferir que homens e mulheres não diferem no modo como enfrentam situações complexas, como a escolha por uma profissão ou carreira.

A título de finalização, muitos estudos têm demonstrado que otimismo e pessimismo possuem efeitos significativos no bem-estar físico e mental dos indivíduos, e também em seu desempenho acadêmico e profissional (Aspinwall \& Taylor, 1992; Carver et al., 2010; Long, 1993; Rashid et al., 2012; Scheier et al., 2001; Yun-Jeong, \& Kevin, 2013). A partir do exposto, infere-se que a Orientação Profissional, ao facilitar o processo de tomada de decisão dos jovens, está associada com o desenvolvimento de sentimentos positivos, que minimizarão as indecisões relacionadas ao processo de tomada de decisão.

\section{Considerações Finais}

O processo de Orientação Profissional é muito importante para que os indivíduos, principalmente os adolescentes que terminam o Ensino Médio, tenham clareza da escolha de uma profissão ou carreira, ou até mesmo da sua inserção no mercado de trabalho (Ginevra et al., 2012). Entretanto, é comum o surgimento de dúvidas e questionamentos sobre si mesmos e sobre o universo tão amplo das profissões. Portanto, o principal objetivo do processo de Orientação Profissional é o auxílio na compreensão das necessidades e características, especialmente as dos adolescentes, com vistas a minimizar a indecisão profissional (Levenfus, 2010; Noronha \& Ambiel, 2008; Primi et al., 2000; Rocha, 2010).

Em relação às limitações do presente estudo, primeiramente destaca-se o número desigual de participantes em relação ao tipo de escola (particular e pública), razão pela qual não foram realizadas análises com essa variável e sim, entre os grupos de estudantes (Grupo 1 - jovens que frequentam o Ensino Médio e Técnico-Profissional conjuntamente, e Grupo 2 - jovens que trabalham e estudam - aprendizes). Ademais, no que se refere ao tipo de público, mais particularmente aos aprendizes, não há muitos estudos com esse público, em levantamento feito nas bases de dados BVS-Psi e SciELO (Scientific Eletronic Library Online). A última consideração a ser feita é que os fatores relacionados à indecisão 
profissional e otimismo e pessimismo podem estar permeados por outras variáveis, necessitando a realização de investigações futuras, tanto com jovens aprendizes, quanto com outros tipos de amostras, para esclarecer essas questões, como por exemplo, as possíveis diferenças entre escolas públicas e particulares, e também as relações com outros construtos. Assim, esta é a agenda de pesquisa que se propõe.

\section{Referências}

Alarcon, G. M., Bowling, N. A., \& Khazon, S. (2013). Great expectations: A meta-analytic examination of optimism and hope. Personality and Individual Differences, 54(7), 821-827. doi:10.1016/j.paid.2012.12.004

Andersson, M. A. (2012). Identity crises in love and at work: Disposicional optimism as a Durable Personal Resource. Social Psychology Quaterly, 75(4), 290-309.

Aspinwall, L. G., \& Taylor, S. E. (1992). Modeling cognitive adaptation: A longitudinal investigation of the impact of individual differences and coping on college adjustament and performance. Journal of Personality and Social Psychology, 61, 755-765.

Bastianello, M. R. (2011). Adaptação e validação do Teste para Avaliação de Otimismo LOT-R e suas relações com autoestima e personalidade (Dissertação de mestrado, Universidade Federal do Rio Grande do Sul, Porto Alegre, RS, Brasil).

Bastianello, M. R., Pacico, J. C., \& Hutz, C. S. (2014). Otimismo, autoestima e personalidade: Estudos de adaptação e validação brasileira do Revised Life Orientation Test (LOT-R). Psico-USF, 19(3), 523-531. doi:10.1590/1413827120140190030

Boehm, J. K., \& Lyubomirsky, S. (2008). Does happiness promote career success? Journal of Career Assessment, 16(1), 101-116. doi:10.1177/1069072707308140

Carvalho, M., \& Taveira, M. C. (2012). A implementação de decisões vocacionais: Revisão da literatura. Revista Brasileira de Orientação Profissional, 13(1), 27-35.

Carver, C. S., \& Scheier, M. F. (1993). On the power of positive thinking: The benefits of being optimistic. Current Directions in Psychological Science, 2(1), 26-30.
Carver, C. S., \& Scheier, M. F. (1999). Optimism. In C. R. Snyder (Ed.), Coping: The psychology of what works (pp. 182-204). New York: Oxford University Press.

Carver, C. S., \& Scheier, M. F. (2005). Optimism. In C. Snyder \& S. Lopez (Eds.), Handbook of Positive Psychology (pp. 751-767). Oxford, UK: Oxford University Press.

Carver, C. S., Scheier, M. F., \& Segerstrom, S. C. (2010). Optimism. Clinical Psychology Review, 30, 879-889. doi:10.1016/j.cpr.2010.01.006

Choi, B. Y., Park, H., Yang, E., Ki Lee, S., Lee, Y., \& Min Lee, S. (2011). Understanding career decision self-eficacy: A meta-analytic approach. Journal of Career Development, 39(5), 443-460. doi:10.1177/0894845311398042

Contreras, F., \& Esguerra, G. (2006). Psicología positiva: una nueva perspectiva en psicología. Diversitas, 2(2), 311-319.

Creed, P. A., Patton, W., \& Bartrum, D. (2002). Multidimension properties of the LOT-R: Effects of optimism and pessimism on career and wellbeing related variables in adolescents. Journal of Career Assessment, 10, 42-61. doi:10.1177/1069072702010001003

Faria, L. C. (2013). Influência da condição de emprego/desemprego dos pais na exploração e indecisão vocacional dos adolescentes. Psicologia: Reflexão \& Crítica, 26(4), 772-779. doi:10.1590/ S0102-79722013000400018

Gati, I., Krauz, M., \& Osipow, S. H. (1996). A taxonomy of difficulties in career decison making. Journal of Counseling Psychology, 43(4), 510-526.

Gati, I., \& Tal, S. (2008). Decision-making models and career guidance. In J. A. Athanasou \& R. V. Esbroeck (Eds.), International Handbook of Career Guidance (pp. 157-185). Sydney, Australia: Springer.

Ginevra, M. C., Nota, L., Soresi, S., \& Gati, I. (2012, June 19). Career decision-making profiles of Italian adolescents. Journal of Carrer Assessment, 1-15. doi:10.1177/1069072712448739

Harpaz-Itay, Y., \& Kaniel, S. (2012). Optimism versus pessimism and academic achievement evaluation. Gifted Education International, 28(3), 267-280. doi:10.1177/0261429411435106

Hartley, S. L. (2009). Career indecision, negative career thoughts, and vocational interest structure 
of first-generation and other college students. Dissertation Abstracts International: Section B: Sciences and Engineering, 70(12-B), 7872.

Hutz, C. S., \& Bardagi, M. P. (2006). Indecisão profissional, ansiedade e depressão na adolescência: A influência dos estilos parentais. Psico-USF, 11(1), 65-73. doi:10.1590/S141382712006000100008

Levenfus, R. S. (2010). Orientação vocacional ocupacional: Abordagem clínica psicológica. In R. S. Levenfus \& D. H. P. Soares (Eds.), Orientação vocacional ocupacional (pp. 117-132). Porto Alegre, RS: Artmed.

Little, K. R. (2012). Teacher academic optimism: A study of teachers' academic optimism and students' perceptions. Dissertation Abstracts International Section A: Humanities and Social Sciences, 72(8-A), 275.

Long, B. C. (1993). Coping strategies of male managers: A prospective analysis of predictors of psychosomatic symptoms and job satisfaction. Journal of Vocational Behavior, 42, 184-199. doi:10.1006/jvbe.1993.1013

Lyubomirsky, S., Tkach, C., \& DiMatteo, M. R. (2006). What are the differences between happiness and self-esteem? Social Indicators Research, 78, 363-404.

Macêdo, O. J. V., Alberto, M. F. P., \& Araujo, A. J. S. (2012). Formação profissional e futuro: Expectativas dos adolescentes aprendizes. Estudos de Psicologia (Campinas), 29(Supl. 1), 779s-787s. doi:10.1590/S0103-166X2012000500014

Magalhães, M. O., Alvarenga, P., \& Teixeira, M. A. P. (2012). Relação entre estilos parentais, instabilidade de metas e indecisão vocacional em adolescentes. Revista Brasileira de Orientação Profissional, 13(1), 15-25.

Melo-Silva, L. L., Lassance, M. C. P., \& Soares, D. H. P. (2004). A orientação profissional no contexto da educação e trabalho. Revista Brasileira de Orientação Profissional, 5(2), 31-52.

Melo-Silva, L., Santos, M. A., Palma, S. P. V., \& Duarte, C. V. (2007). Felicidade sob medida: Expressão da ideologia no processo de orientação profissional. In D. T. R. Barros, M. T. Lima, \& R. Escalda (Eds.), Escolha e inserção profissionais: Desafios para indivíduos, famílias e instituições: Vol. 3. Orientação profissional: Teoria e técnica (pp. 157-179). São Paulo, SP: Vetor.
Ministério do Trabalho e Emprego. (2000). Informativo Aprendizagem. Recuperado em http:// www3.mte.gov.br/politicas_juventude/aprendizagem_default.asp

Noronha, A. P. P., \& Ambiel, R. A. M. (2008). Estudo correlacional entre Escala de Aconselhamento Profissional (EAP) e Self-Directed Search (SDS). Interação em Psicologia, 12(1), 21-33.

Oliveira, C. M. R., \& Neiva, K. M. C. (2013). Orientação vocacional/profissional: Avaliação de um projeto piloto para estudantes da educação profissional. Revista Brasileira de Orientação Profissional, 14(1), 133-143.

Primi, R., Munhoz, A. M. H., Bighetti, C. A., Porto, E. D. N., Pellegrini, M. C. K., \& Moggi, M. A. (2000). Desenvolvimento de um Inventário de Levantamento das Dificuldades da Decisão Profissional. Psicologia: Reflexão $e$ Crítica, 13(3), 451-463. doi:10.1590/S010279722000000300013

Proyer, R. T., Sidler, N., Weber, M., \& Ruch, W. (2012). A multi-method approach to studying the relationship between character strengths and vocational interests in adolescents. International Journal for Educational and Vocational Guidance, 12(2), 141-157. doi:10.1007/s10775012-9223-x

Rashid, S., Arif, M. I., \& Hussain, M. (2012). An investigation into relationship among stress, optimism and life satisfaction of adolescents. International Journal of Social Sciences \& Educational, 3, 221-231.

Rocha, M. C. S. (2010). Projeto de carreira, plano de vida: Passos para um gerenciamento de vida profissional e pessoal. In R. S. Levenfus \& D. H. P. Soares (Eds.), Orientação vocacional ocupacional (pp. 82-91). Porto Alegre, RS: Artmed.

Seligman, M. E. P. (2002). Positive psychology, positive prevention, and positive therapy. In C. R. Snyder \& S. J. Lopez (Eds.), Handbook of Positive Psychology (pp. 3-9). New York: Oxford University Press.

Scheier, M. F., \& Carver, C. S. (1985). Optimism, coping, and health - Assessment and implications of generalized outcome expectancies. Health Psychology, 4(3), 219-247.

Scheier, M. F., \& Carver, C. S. (1993). On the power of positive thinking: The benefits of being optimistic. Current Directions in Psychological Science, 2, 26-30. 
Scheier, M. F., Carver, C. S., \& Bridges, M. W. (1994). Distinguishing optimism from neuroticism (and trait anxiety, self-mastery, and selfesteem): A reevaluation of the Life Orientation Test. Journal of Personality and Social Psychology, 67, 1063-1078.

Scheier, M. F., Carver, C. S., \& Bridges, M. W. (2001). Optimism, pessimism, and psychological well-being. In E. C. Chang (Ed.), Optimism and pessimism: Implications for theory, research, and practice (pp. 189-216). Washington, DC: American Psychological Association.

Shane, J. L., \& Snyder, C. R. (2003). Positive psychological assessment: $A$ handbook of models and measures. Washington, DC: American Psychological Association.

Sobrosa, G. M. R., Camerin, C., Santos, A. S., \& Dias, A. C. G. (2012). Considerações acerca da inserção profissional de jovens do ensino médio. $M u$ danças - Psicologia da Saúde, 20(1-2), 41-49. doi:10.15603/2176-1019/mud.v20n1-2p41-49
Stoltz, K. B., \& Young, T. L. (2012, August 17). Applications of motivational interviewing in career counseling: Facilitating career transition. Journal of Career Development, 1-18. doi: $10.1177 / 0894845312455508$

Viloria, A. R., Yáñez, M. S. S., \& Vañó, A. C. (2010). El optimismo - aplicaciones educativas. In A. C. Vañó (Ed.), Aplicaciones educativas de la psicología positiva (pp. 161- 184). Alicante, España: Generalitat Valenciana.

Yun-Jeong, S., \& Kevin, K. R. (2013). Cross-cultural comparison of the effects of optimism, intrinsic motivation and family relations on vocational identity. The Career Development Quarterly, 61, 141-160.
Recebido: 21/05/2014

$1^{a}$ revisão: 04/11/2014

$2^{a}$ revisão: 03/02/2015

Aceite final: 10/02/2015 